\title{
CRITICAL ELASTIC CONSTANTS AND VISCOSITIES ABOVE A NEMATIC-SMECTIC A TRANSITION OF SECOND ORDER
}

\author{
F. JÄHNIG $\left({ }^{*}\right)$ \\ Physique de la Matière Condensée, Collège de France, 11, place Marcelin-Berthelot, \\ 75231 Paris Cedex 05, France \\ and \\ F. BROCHARD
}

Laboratoire de Physique des Solides (**), Université de Paris-Sud, 91405 Orsay, France

(Reçu le 17 octobre 1973, révisé le 14 novembre 1973)

\begin{abstract}
Résumé. - Les fluctuations du paramètre d'ordre à une transition nématique-smectique $\mathrm{A}$ du $2^{\mathrm{e}}$ ordre créent des singularités de certaines viscosités et constantes élastiques en phase nématique. Le comportement critique des constantes élastiques à déjà été calculé par P. G. de Gennes dans le régime hydrodynamique $q \xi \ll 1$. En partant des mêmes hypothèses et en utilisant comme outil de travail la théorie de la réponse linéaire et le théorème de fluctuation-dissipation, on montre que certaines viscosités divergent comme $\left(T-T_{\mathrm{c}}\right)^{-0,33}$. Notre calcul s'applique aussi dans la région critique $q \xi \gg 1$, où l'on trouve que la largeur du spectre des fluctuations du directeur est proportionnelle à $q^{3 / 2}$. Ce résultat confirme une prédiction récente de $\mathrm{F}$. Brochard basée sur un argument de similarité dynamique.
\end{abstract}

\begin{abstract}
Abstraet. - The fluctuations of the local order parameter above a second order nematic-smetic A phase transition give rise to singularities in the elastic constants and the viscosities of a nematic. In the hydrodynamic regime $q \xi \ll 1$, the critical behaviour of the elastic constants has already been calculated by de Gennes. Based on the same assumptions, we derive a singularity of the critical viscosities proportional to $\left(T-T_{\mathrm{c}}\right)^{-0.33}$. As theoretical framework we use linear response theory and the fluctuation-dissipation theorem. We treat also the general case of arbitrary wave numbers. In the critical regime $q \xi \gg 1$, we find a critical spectrum of the director modes $\omega_{\mathrm{S}} \sim q^{3 / 2}$, in agreement with a recent suggestion of $F$. Brochard based on a dynamical scaling argument.
\end{abstract}

1. Introduction. - Recently there has been considerable interest in the nematic-smectic $\mathrm{A}[\mathrm{N}-\mathrm{A}]$ phase transition as it may occur as a transition of second order. In this case the fluctuations of the order parameter show up in strong pretransitional effects just above the transition temperature $T_{\mathrm{c}}$. Since the smectic A phase does not accept twist and bend deformations one expects the associated Frank elastic constants $K_{2}$ and $K_{3}$ to show a critical behaviour. A divergence $K_{2} \sim K_{3} \sim\left(T-T_{\mathrm{c}}\right)^{-0.66}$ was predicted theoretically by de Gennes [1] and confirmed recently by three independent measurements [2]-[4]. De Gennes

$\left(^{*}\right)$ Stipendiat der Deutschen Forschungsgemeinschaft. On leave from the Physik-Department der Technischen Universität München, Germany.

$\left({ }^{* *}\right)$ Associated with Centre national de la Recherche scientifique. started with a definition of the order parameter of the smectic A phase as a complex quantity $\psi(\mathbf{r})$, the modulus of which determines the density of the layers and the phase determines the position of the layers. For a given configuration of $\psi(\mathbf{r})$ the free energy of the system is given by a Ginzburg-Landau type expression, i. e. the coupling of the director field $\mathbf{n}(\mathbf{r})$ to the order parameter has been taken into account in analogy to the coupling of the magnetic field to the order parameter in a superconductor. Calculating the unrestricted free energy as an average over all configurations of $\psi(\mathbf{r})$ due to static fluctuations one obtains the critical contributions to the elastic constants, in full analogy to the critical diamagnetic susceptibility above the superconducting transition [5].

The onset of superconductivity is marked in addition by a critical increase of the electrical conductivity. 
The reason for this critical dissipation lies in the dynamic fluctuations of the order parameter which slow down if $T_{\mathrm{c}}$ is approached. Somewhat similarly, above a nematic-smectic A transition the slowing down of the order parameter fluctuations leads to a critical contribution to viscosities. This contribution is the main aim of the present paper. We calculate it using linear response theory as it was applied to superconductors by $\mathrm{H}$. Schmidt [6]. The response functions are related to the dynamical fluctuations by means of the fluctuation-dissipation theorem.

We start in section 2 with a presentation of the free energy and the dynamical fluctuations of the order parameter. For the critical exponents entering we take the values suggested by de Gennes [1] and F. Brochard [7] on assuming that the N-A transition is thermodynamically similar to the $\lambda$-transition in helium, rather than to the critical point of a superconductor where mean field theory holds. In section 3, following the Orsay Liquid Crystal Group [8], we introduce the nematic molecular field $\mathbf{h}(\mathbf{r}, t)$ as the variable conjugate to the director $\mathbf{n}(\mathbf{r}, t)$. We are interested in the thermal average $\langle\mathbf{h}(\mathbf{r}, t)\rangle$ as a response to a small external perturbation $\delta \mathbf{n}^{\text {ext }}\left(\mathbf{r}^{\prime}, t^{\prime}\right)$. Using linear response theory and the fluctuation- dissipation theorem we derive the corresponding director response function in terms of the fluctuations of the order parameter. In section 4 we show that this expression fulfils the general conditions imposed by the invariance properties of the system. We then apply, in section 5, the result obtained for the response function to the determination of the critical part of the Frank elastic constants, not only in the hydrodynamic regime where we recover de Gennes' results but also in the critical regime. The critical part of the twist viscosity $\gamma_{1}$ is calculated in section 6 . The other viscosities of an incompressible nematic are treated in section 7 by relating them to $\gamma_{1}$ using standard Kubo relations.

2. Thermal fluctuations. - The free energy for a system with a nematic and a smectic A phase may be expressed [1] in terms of the local smectic order parameter $\psi(\mathbf{r}, t)$ and the local director

$$
\begin{gathered}
\mathbf{n}(\mathbf{r}, t)=\mathbf{n}_{0}+\delta \mathbf{n}(\mathbf{r}, t) \\
F-F_{0}=\int \mathrm{d} \mathbf{r}\left(F_{\mathrm{S}}+F_{\mathrm{N}}\right)
\end{gathered}
$$

with

$$
F_{\mathrm{S}}=A|\psi(\mathbf{r}, t)|^{2}+\frac{1}{2} B|\psi(\mathbf{r}, t)|^{4}+\frac{1}{2 M_{\mathrm{T}}}\left|\left(\nabla_{\perp}-i q_{\mathrm{S}} \delta \mathbf{n}(\mathbf{r}, t)\right) \psi(\mathbf{r}, t)\right|^{2}+\frac{1}{2 M_{\mathbf{V}}}\left|\nabla_{\|} \psi(\mathbf{r}, t)\right|^{2}
$$

and

$$
F_{\mathbf{N}}=\frac{1}{2}\left\{K_{1}(\operatorname{div} \delta \mathbf{n}(\mathbf{r}, t))^{2}+K_{2}\left(\mathbf{n}_{0} \cdot \operatorname{rot} \delta \mathbf{n}(\mathbf{r}, t)\right)^{2}+K_{3}\left(\mathbf{n}_{0} \wedge \operatorname{rot} \delta \mathbf{n}(\mathbf{r}, t)\right)^{2}\right\}
$$

The smectic energy contribution $F_{\mathrm{S}}$ has the GinzburgLandau form, familiar for charged superfluids. The signs parallel and perpendicular are defined with respect to the preferred axis $\mathbf{n}_{0}$, which in the following is taken as the $z$ direction, therefore $\delta n_{z}=0$. The constant $q_{\mathrm{s}}$ is related to the interlayer distance $d$ by $q_{\mathrm{s}}=2 \pi / d$, the $K_{1,2,3}$ are the Frank elastic constants for splay, twist, and bend deformations, respectively.

Fourier transformation of eq. (2.2) and application of the equipartition theorem yield for the static fluctuations of the order parameter, for $T>T_{\mathrm{c}}$,

$$
\begin{aligned}
\left\langle\psi^{*}(\mathbf{K}) \psi\left(\mathbf{K}^{\prime}\right)\right\rangle & = \\
= & \left\langle|\psi(\mathbf{K})|^{2}\right\rangle(2 \pi)^{3} \delta\left(\mathbf{K}-\mathbf{K}^{\prime}\right)
\end{aligned}
$$

with (we shall use Boltzmann's constant as unit, $\left.K_{\mathrm{B}}=1\right)$

$$
\left\langle|\psi(\mathbf{K})|^{2}\right\rangle=\frac{T}{A\left(1+K_{\perp}^{2} \xi_{\perp}^{2}+K_{z}^{2} \xi_{\|}^{2}\right)} .
$$

The coherence lengths $\xi_{\|}$and $\xi_{\perp}$ are defined by

$$
\begin{aligned}
& \xi_{\|}=\left(2 A M_{\mathrm{V}}\right)^{-1 / 2} \\
& \xi_{\perp}=\left(2 A M_{\mathrm{T}}\right)^{-1 / 2} .
\end{aligned}
$$

As mentioned above, it was supposed by de Gennes [1] that the N-A transition is thermodynamically similar to the $\lambda$-transition in helium, an uncharged superfluid. Therefore he suggested that

$$
\xi(T) \sim\left(T-T_{\mathrm{c}}\right)^{-0.66}
$$

and we shall follow this assumption, which holds on static scaling theory.

We keep the assumption of an Ornstein-Zernike form for the static fluctuations of the order parameter, as the deviations seem to be very small $(\eta=0.04)$.

To obtain the dynamical fluctuations we assume a simple relaxation behaviour of the order parameter. 
In Fourier space with

$$
\psi(\mathbf{r}, t)=\int \frac{\mathrm{d} \mathbf{K} \mathrm{d} \Omega}{(2 \pi)^{4}} \psi(\mathbf{K}, \Omega) \mathrm{e}^{-i \Omega t+i \mathbf{K r}}
$$

we write

$$
(-i \Omega+\Gamma(\mathbf{K})) \psi(\mathbf{K}, \Omega)=0
$$

with the following expression for the frequency factor $\Gamma(\mathbf{K})$

$$
\frac{1}{\Gamma(\mathbf{K})}=\frac{\tau_{\mathrm{m}}}{\left(1+K_{\perp}^{2} \xi_{\perp}^{2}+K_{z}^{2} \xi_{\|}^{2}\right)^{x}}
$$

Applying dynamical scaling arguments F. Brochard [7] found for the relaxation time $\tau_{\mathrm{m}}$ of the order parameter

$$
\tau_{\mathrm{m}}(T) \sim \xi^{3 / 2}(T)
$$

and

$$
x=\frac{3}{4}
$$

Eq. (2.9) is constructed to give a temperature independent expression in the critical limit $K \xi \gg 1$

$$
\Gamma(K) \sim K^{3 / 2} \text {. }
$$

The frequency spectrum of the order parameter fluctuations is easily obtained from eq. (2.8) as

$$
\begin{aligned}
& \left\langle\psi^{*}(\mathbf{K}, \Omega) \psi\left(\mathbf{K}, \Omega^{\prime}\right)\right\rangle= \\
& =2\left\langle|\psi(\mathbf{K})|^{2}\right\rangle \frac{\Gamma(\mathbf{K})}{\Omega^{2}+\Gamma^{2}(\mathbf{K})} 2 \pi \delta\left(\Omega-\Omega^{\prime}\right) .
\end{aligned}
$$

It should be mentioned that in the critical region, there may be a propagating contribution to this spectrum. As this would only modify slightly the numerical constants of our results, we neglect it in the following.

3. The response function. - The molecular field $\mathbf{h}$ acting on the director has been introduced by the Orsay Liquid Crystal Group [8]. The formal definition of $\mathbf{h}$ is obtained from the variation of the free energy $F$ with respect to the director

$$
\delta F=-\int \mathrm{d} \mathbf{r} \mathbf{h}^{t}(\mathbf{r}, t) \delta \mathbf{n}(\mathbf{r}, t)
$$

The fluctuating part $\tilde{\mathbf{h}}$ of $\mathbf{h}^{t}=\mathbf{h}+\tilde{\mathbf{h}}$ results from the smectic part $F_{S}$ of the free energy as

$$
\begin{aligned}
& \tilde{\mathbf{h}}(\mathbf{r}, t)= \\
& =-\frac{i q_{\mathrm{S}}}{2 M_{\mathrm{T}}}\left(\psi^{*}(\mathbf{r}, t) \nabla_{\perp} \psi(\mathbf{r}, t)-\psi(\mathbf{r}, t) \nabla_{\perp} \psi^{*}(\mathbf{r}, t)\right)- \\
& \quad-\frac{q_{\mathrm{S}}^{2}}{M_{\mathrm{T}}} \psi^{*}(\mathbf{r}, t) \psi(\mathbf{r}, t) \delta \mathbf{n}(\mathbf{r}, t) .
\end{aligned}
$$

We now want to determine $\langle\tilde{\mathbf{h}}(\mathbf{r}, t)\rangle_{\text {n.e. }}$, the thermal average of the fluctuating molecular field if a small external perturbation $\delta \mathbf{n}^{\text {ext }}\left(\mathbf{r}^{\prime}, t^{\prime}\right)$ is applied to the system. According to the general lines of linear response theory we obtain in Fourier space

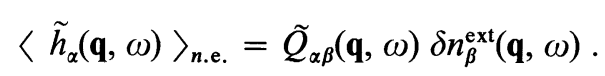

The response function $\widetilde{Q}_{\alpha \beta}$ is the sum of two essentially different terms

$$
\widetilde{Q}_{\alpha \beta}(\mathbf{q}, \omega)=Q_{\alpha \beta}^{\mathrm{s}}+Q_{\alpha \beta}^{\mathrm{d}}(\mathbf{q}, \omega) .
$$

The static term

$$
Q_{\alpha \beta}^{\mathrm{s}}=-\frac{q_{\mathrm{S}}^{2}}{M_{\mathrm{T}}}\left\langle|\psi|^{2}\right\rangle \delta_{\alpha \beta}
$$

arising from the second term of $\tilde{\mathbf{h}}$, eq. (3.2), is determined by the mean value in space and time of the thermal fluctuations of the order parameter. The second term $Q_{\alpha \beta}^{\mathrm{d}}$ arises from the dynamical fluctuations of the order parameter in equilibrium. Using the fluctuation-dissipation theorem (for classical systems) the imaginary part of $Q_{\alpha \beta}^{\mathrm{d}}$ is given by

$$
Q_{\alpha \beta}^{\mathrm{d} \prime \prime}(\mathbf{q}, \omega)=\frac{\omega}{2 T}\left\langle\tilde{h}_{\alpha}(\mathbf{q}, \omega) \tilde{h}_{\beta}(-\mathbf{q},-\omega)\right\rangle .
$$

According to the dispersion relations the real part can be obtained as

$$
Q_{\alpha \beta}^{\mathrm{d}^{\prime}}(\mathbf{q}, \omega)=f \frac{\mathrm{d} \omega^{\prime}}{\pi} \frac{Q_{\alpha \beta}^{\mathrm{d}^{\prime \prime}}\left(\mathbf{q}, \omega^{\prime}\right)}{\omega^{\prime}-\omega}
$$

Neglecting the fluctuations of the director we can identify $\delta \mathbf{n}$ with $\delta \mathbf{n}^{\text {ext }}$. Then only the first term of $\tilde{\mathbf{h}}$, eq. (3.2), contributes to the response function $Q_{\alpha \beta}^{d}$

$$
Q_{\alpha \beta}^{\mathrm{d}^{\prime \prime}}(\mathbf{q}, \omega)=\frac{\omega}{2 T}\left\langle\tilde{h}_{1 \alpha}(\mathbf{q}, \omega) \tilde{h}_{1 \beta}(-\mathbf{q},-\omega)\right\rangle
$$

with

$$
\widetilde{h}_{1 \alpha}(\mathbf{q}, \omega)=\frac{q_{\mathrm{s}}}{M_{\mathrm{T}}} \int \frac{\mathrm{d} \mathbf{K} \mathrm{d} \Omega}{(2 \pi)^{4}} K_{\text {上 }} \psi *\left(\mathbf{K}-\frac{\mathbf{q}}{2}, \Omega-\frac{\omega}{2}\right) \psi\left(\mathbf{K}+\frac{\mathbf{q}}{2}, \Omega+\frac{\omega}{2}\right)
$$

which yields

$$
\begin{aligned}
& Q_{\alpha \beta}^{\mathrm{d}^{\prime \prime}}(\mathbf{q}, \omega)=\frac{\omega q_{\mathrm{S}}^{2}}{2 T M_{\mathrm{T}}^{2}} \int \frac{\mathrm{d} \mathbf{K}_{1} \mathrm{~d} \mathbf{K}_{2} \mathrm{~d} \Omega_{1} \mathrm{~d} \Omega_{2}}{(2 \pi)^{8}} K_{1 \perp \alpha} K_{2 \perp \beta} \\
& \quad\left\langle\psi^{*}\left(\mathbf{K}_{1}-\frac{\mathbf{q}}{2}, \Omega_{1}-\frac{\omega}{2}\right) \psi\left(\mathbf{K}_{1}+\frac{\mathbf{q}}{2}, \Omega_{1}+\frac{\omega}{2}\right) \psi^{*}\left(\mathbf{K}_{2}+\frac{\mathbf{q}}{2}, \Omega_{2}+\frac{\omega}{2}\right) \psi\left(\mathbf{K}_{2}-\frac{\mathbf{q}}{2}, \Omega_{2}-\frac{\omega}{2}\right)\right\rangle .
\end{aligned}
$$


We now apply a decoupling approximation between the real and the imaginary part of the order parameter. This has been successfully introduced by Ferrell [17] in the case of superfluid He, ferro- and antiferromagnets, and binary mixtures. For these systems, mean field theory does not hold (but $\eta$ has to be negligible).

Using eq. (2.4) and (2.12) we arrive at the following expression

$$
\begin{aligned}
Q_{\alpha \beta}^{\mathrm{d}^{\prime \prime}}(\mathbf{q}, \omega)=\frac{\omega q_{\mathrm{S}}^{2}}{T M_{\mathrm{T}}^{2}} \int \frac{\mathrm{d} \mathbf{K}}{(2 \pi)^{3}} K_{\perp \alpha} K_{\perp \beta}\left\langle\left|\psi\left(\mathbf{K}-\frac{\mathbf{q}}{2}\right)\right|^{2}\right\rangle\left\langle\left|\psi\left(\mathbf{K}+\frac{\mathbf{q}}{2}\right)\right|^{2}\right\rangle \dot{\times} & \times \frac{\Gamma\left(\mathbf{K}-\frac{\mathbf{q}}{2}\right)+\Gamma\left(\mathbf{K}+\frac{\mathbf{q}}{2}\right)}{\omega^{2}+\left[\Gamma\left(\mathbf{K}-\frac{\mathbf{q}}{2}\right)+\Gamma\left(\mathbf{K}+\frac{\mathbf{q}}{2}\right)\right]^{2}} .
\end{aligned}
$$

By means of the dispersion relation eq. (3.7) we finally obtain the full response function

$$
\begin{aligned}
Q_{\alpha \beta}^{\mathrm{d}}(\mathbf{q}, \omega)=\frac{q_{\mathrm{S}}^{2}}{T M_{\mathbf{T}}^{2}} \int \frac{\mathrm{d} \mathbf{K}}{(2 \pi)^{3}} \mathbf{K}_{\perp \alpha} \mathbf{K}_{\perp \beta}\left\langle\left|\psi\left(\mathbf{K}-\frac{\mathbf{q}}{2}\right)\right|^{2}\right\rangle\left\langle\left|\psi\left(\mathbf{K}+\frac{\mathbf{q}}{2}\right)\right|^{2}\right\rangle \times & \Gamma \frac{\Gamma\left(\mathbf{K}-\frac{\mathbf{q}}{2}\right)+\Gamma\left(\mathbf{K}+\frac{\mathbf{q}}{2}\right)}{-i \omega+\Gamma\left(\mathbf{K}-\frac{\mathbf{q}}{2}\right)+\Gamma\left(\mathbf{K}+\frac{\mathbf{q}}{2}\right)}
\end{aligned}
$$

The part $\mathbf{h}(\mathbf{r}, t)$ of the total molecular field, eq. (3.1), resulting from the variation of $F_{\mathrm{N}}$, eq. (2.1), is the noncritical hydrodynamic response. Generalization to the case with dissipation (due to rotational motion only) yields

$$
\begin{aligned}
& h_{x}(\mathbf{q}, \omega)=i \omega \gamma_{1} \delta n_{x}^{\mathrm{ext}}(\mathbf{q}, \omega)-\left(K_{1} q_{x}^{2}+K_{2} q_{y}^{2}+K_{3} q_{z}^{2}\right) \delta n_{x}^{\mathrm{ext}}(\mathbf{q}, \omega)+\left(K_{2}-K_{1}\right) q_{x} q_{y} \delta n_{y}^{\mathrm{ext}}(\mathbf{q}, \omega) \\
& h_{y}(\mathbf{q}, \omega)=i \omega \gamma_{1} \delta n_{y}^{\mathrm{ext}}(\mathbf{q}, \omega)-\left(K_{1} q_{y}^{2}+K_{2} q_{x}^{2}+K_{3} q_{z}^{2}\right) \delta n_{y}^{\mathrm{ext}}(\mathbf{q}, \omega)+\left(K_{2}-K_{1}\right) q_{x} q_{y} \delta n_{x}^{\mathrm{ext}}(\mathbf{q}, \omega),
\end{aligned}
$$

$\gamma_{1}$ being the twist viscosity.

The total response $\langle\widetilde{\mathbf{h}}\rangle_{n . e .}+\mathbf{h}$ may be written in the form of the purely hydrodynamic eq. (3.13) on substituting the $K_{i}$ 's and $\gamma_{1}$ by

$$
K_{i}^{t}=K_{i}+\tilde{K}_{i}, \quad \gamma_{1}^{t}=\gamma_{1}+\tilde{\gamma}_{1},
$$

where $\tilde{K}_{i}$ and $\tilde{\gamma}_{1}$ represent the critical contributions to the hydrodynamic parameters of the nematic phase due to thermal fluctuations of the smectic order parameter above the $\mathrm{N}-\mathrm{A}$ transition.

4. Smectic gauge invariance. - Before applying eq. (3.12) to the calculation of the critical contribution to the elastic and viscous parameters we want to discuss a general constraint on the response function. Our system is invariant, for $T>T_{\mathrm{c}}$, under a simultaneous local rotation of the layers and the director (except for the noncritical splay term in $F_{\mathrm{N}}$ ). This property corresponds to the invariance under a gauge transformation of a superconductor, above the superconducting transition, and will therefore be called smectic gauge invariance. The transformation is represented by

and

$$
\psi(\mathbf{r})=\psi^{\prime}(\mathbf{r}) \mathrm{e}^{-i \Phi(\mathbf{r})}
$$

$$
\delta \mathbf{n}(\mathbf{r})=\delta \mathbf{n}^{\prime}(\mathbf{r})+\nabla \chi(\mathbf{r}) .
$$

The invariance of the free energy or the molecular field expressions, eq. (2.2) or (3.2), under this transformation imposes the condition

$$
\nabla_{\perp} \Phi(\mathbf{r})=-q_{\mathbf{s}} \nabla_{\perp} \chi(\mathbf{r}) .
$$

We see that this transformation may be used to eliminate the longitudinal part of the director $\delta \mathbf{n}$ from the coupling term in $F_{\mathbf{S}}$. We separate

$$
\delta \mathbf{n}(\mathbf{r})=\delta \mathbf{n}_{l}(\mathbf{r})+\delta \mathbf{n}_{t}(\mathbf{r})
$$

with

$$
\nabla \wedge \delta \mathbf{n}_{l}(\mathbf{r})=0 \text { and } \nabla . \delta \mathbf{n}_{t}(\mathbf{r})=0
$$

(The condition $\delta n_{z}=\delta n_{l z}+\delta n_{t z}=0$ fixes the direction of $\delta \mathbf{n}_{t}$ in the plane $\perp \mathbf{q}$, so $\delta \mathbf{n}$ still has two degrees of freedom). Then eq. (4.1) yields

$$
\delta \mathbf{n}^{\prime}(\mathbf{r})=\delta \mathbf{n}_{t}(\mathbf{r}) \text { and } \nabla \chi(\mathbf{r})=\delta \mathbf{n}_{l}(\mathbf{r}) .
$$

$F_{\mathrm{S}}$ may therefore be expressed in terms of $\psi^{\prime}(\mathbf{r})$ and the transverse part of the director $\delta \mathbf{n}_{t}(\mathbf{r})$. This turns out to be important for the calculation of the response function including thermal fluctuations of the director.

In the ordered phase, we may set $\Phi(\mathbf{r})=q_{\mathrm{s}} u_{z}(\mathbf{r})$, where $u_{z}$ is the displacement of the layers in the $z$ direction, and take $\psi^{\prime}(\mathbf{r})$ as real. Then the smectic 
gauge invariance condition eq. (4.2) is

$$
\nabla_{\perp} u_{z}(\mathbf{r})=-\delta \mathbf{n}_{l}(\mathbf{r}) .
$$

This condition may be used to transform the splay term in $F_{\mathrm{N}}$ into the second order elastic term

$$
\frac{1}{2} K_{1}\left(\frac{\partial^{2} u_{z}}{\partial x^{2}}+\frac{\partial^{2} u_{z}}{\partial y^{2}}\right)^{2}
$$

The smectic gauge invariance has also to be fulfilled by the static response $\langle\tilde{\mathbf{h}}(\mathbf{q}, 0)\rangle_{\text {n.e. }}$. The transformation eq. (4.1) applied to eq. (3.3) yields

or

$$
q_{\beta} \tilde{Q}_{\alpha \beta}(\mathbf{q}, 0)=0
$$

$$
\widetilde{Q}^{l}(\mathbf{q}, 0)=0
$$

where $\tilde{Q}^{l}$ means the longitudinal part of the response function. This condition states that the static response is transverse.

Above $T_{\mathrm{c}}$, in the limit $q \rightarrow 0$, this yields also

$$
\widetilde{Q}^{t}(0,0)=\widetilde{Q}^{l}(0,0)=0 \text {. }
$$

Our response function actually fulfils this condition. As shown in Appendix A, the dynamical part $Q_{\alpha \beta}^{\mathrm{d}}$ $(\mathbf{q}, 0)$, eq. (3.12), of the response function yields in the static limit

$$
q_{\beta} Q_{\alpha \beta}^{\mathrm{d}}(\mathbf{q}, 0)=-q_{\beta} Q_{\alpha \beta}^{\mathrm{s}}
$$

and thereforee eq. (4.4) is fulfilled.

5. The elastic constants. - To calculate the critical part of the Frank elastic constants we derive the real part of the response function $Q^{\mathrm{d}}$ in the static limit from eq. (3.12). We treat only the case $\alpha=\beta=x$, as the other cases do not yield any further information, and get

$$
\begin{aligned}
& Q_{x x}^{\mathrm{d}^{\prime}}(\mathbf{q}, 0)=\frac{q_{\mathrm{S}}^{2}}{T M_{\mathrm{T}}^{2}} \int \frac{\mathrm{d} \mathbf{K}}{(2 \pi)^{3}} K_{x}^{2} \times \\
& \quad \times\left\langle\left|\psi\left(\mathbf{K}-\frac{\mathbf{q}}{2}\right)\right|^{2}\right\rangle\left\langle\left|\psi\left(\mathbf{K}+\frac{\mathbf{q}}{2}\right)\right|^{2}\right\rangle .
\end{aligned}
$$

Inserting eq. (2.5) we obtain

$$
\begin{aligned}
& Q_{x x}^{\mathrm{d}^{\prime}}(\mathbf{q}, 0)=\frac{4 T q_{\mathrm{S}}^{2}}{(2 \pi)^{3} \xi_{\|}} \times \\
& \times \int \mathrm{d} \mathbf{P} \frac{P_{x}^{2}}{\left[1+\left(\mathbf{P}+\frac{\hat{\mathbf{q}}}{2}\right)^{2}\right]\left[1+\left(\mathbf{P}-\frac{\hat{\mathbf{q}}}{2}\right)^{2}\right]}
\end{aligned}
$$

where we have substituted

$$
\xi_{\perp} \mathbf{q}_{\perp} \equiv \hat{\mathbf{q}}_{\perp} \quad \text { and } \quad \xi_{\|} q_{z} \equiv \hat{q}_{z}
$$

First we want to treat the hydrodynamic regime $q \xi \ll 1$, where we have to determine the lowest order term of $Q_{x x}^{\mathrm{d}^{\prime}}(\mathbf{q}, 0)$ in an expansion in powers of $q$. As $Q_{x x}^{\mathrm{d}^{\prime}}(0,0)$ is compensated by $Q_{x x}^{\mathrm{s}^{\prime}}$, eq. (4.5), we are left with a term quadratic in $q$

$$
\begin{aligned}
Q_{x x}^{\prime}(\mathbf{q}, 0) & =-\frac{4 T q_{\mathrm{s}}^{2}}{(2 \pi)^{3} \xi_{\|}} \times \\
& \times \int \mathrm{d} \mathbf{P} \frac{P_{x}^{2}}{\left(1+P^{2}\right)^{3}}\left[\frac{1}{2} \hat{q}^{2}-(\mathbf{P} \cdot \hat{\mathbf{q}})^{2}\right] .
\end{aligned}
$$

The integration can easily be done to give

$$
Q_{x x}^{\prime}(\mathbf{q}, 0)=-\frac{T q_{\mathrm{S}}^{2}}{24 \pi \xi_{\|}}\left(\xi_{\perp}^{2} q_{y}^{2}+\xi_{\|}^{2} q_{z}^{2}\right) .
$$

Comparing the corresponding response $\left\langle\widetilde{h}_{x}(\mathbf{q}, \omega)\right\rangle_{\text {n.e. }}$ with eq. (3.13) and using the definitions eq. (3.14) we find the following result for the critical part of the elastic constants

$$
\begin{aligned}
& \tilde{K}_{1}=0 \\
& \tilde{K}_{2}=\frac{T q_{\mathrm{S}}^{2}}{24 \pi} \frac{\xi_{\perp}^{2}}{\xi_{\|}}=\frac{\pi T}{6 \mathrm{~d}^{2} M_{\mathrm{T}}} \sqrt{\frac{M_{\mathrm{V}}}{2 A}} \\
& \tilde{K}_{3}=\frac{T q_{\mathrm{S}}^{2}}{24 \pi} \xi_{\|}=\frac{\pi T}{6 d^{2}} \sqrt{\frac{1}{2 A M_{\mathrm{V}}}} .
\end{aligned}
$$

The noncritical behaviour of $K_{1}$ expresses the' fact that the static response due to fluctuations is transverse. The result for $\widetilde{K}_{2}$ and $\widetilde{K}_{3}$ agrees with de Gennes' [1] (except for a factor $1 / \sqrt{2}$ which is missing in his expressions)

$$
\tilde{K}_{2}(T) \sim \tilde{K}_{3}(T) \sim \xi(T) \sim\left(T-T_{\mathrm{c}}\right)^{-0.66} .
$$

The noncritical behaviour of the splay elastic constant $K_{1}$, eq. ( $5.5 a$ ), has been verified by Cheung, Meyer, and Gruler [2]. The critical temperature dependence of the twist elastic constant $\widetilde{K}_{2}$, eq. (5.6), has been confirmed by Delaye, Ribotta, and Durand [4] using quasielastic light scattering, and the critical behaviour of the bend elastic constant $\tilde{K}_{3}$ has been verified by Cheung et al. [2], and by Léger [3] applying the magnetic field induced Frederiks transition.

Next we want to treat the general case of arbitrary values of $q \xi$. As static scaling holds for our expressions for the static fluctuations of the order parameter, eq. (2.5), eq. (5.2) for the static response function can still be used. The integration in the general case is a little tricky, we give it in Appendix B. The result is

$$
\begin{aligned}
& Q_{x x}^{\prime}(\mathbf{q}, 0)=-\frac{T q}{4 \pi \xi_{\|}} \times \\
& \quad \times\left[\left(1+\left(\frac{\hat{q}}{2}\right)^{2}\right) \frac{2}{\hat{q}} \arcsin \left(\frac{\hat{q} / 2}{\sqrt{1+(\hat{q} / 2)^{2}}}\right)-1\right]
\end{aligned}
$$


where we have put $q_{x}=0$ as we know already that the static response is transverse, and therefore

$$
\hat{q}=\sqrt{\hat{q}_{y}^{2}+\hat{q}_{z}^{2}} .
$$

The $q$ dependence of $Q_{x x}^{\prime}(\mathbf{q}, 0)$ is shown in figure 1 .

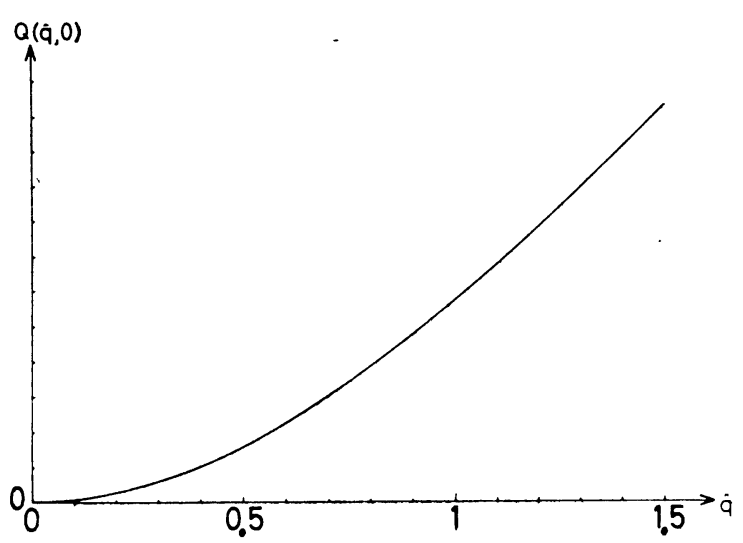

FIG. 1. - Wave number dependence of the static response function.

In the hydrodynamic limit $q \xi \ll 1$, eq. (5.7) yields the above result, eq. (5.4). In the critical limit $q \xi \gg 1$, eq. (5.7) yields

$$
Q_{x x}^{\prime}(\mathbf{q}, 0)=-\frac{T q_{\mathrm{S}}^{2}}{16} \sqrt{\frac{M_{\mathrm{V}}}{M_{\mathrm{T}}} q_{y}^{2}+q_{z}^{2}} .
$$

This expression shows no critical temperature dependence as required by static scaling. If one wants to keep the definition of an elastic constant in the critical limit, either for $q_{y}=0$ or $q_{z}=0$, e. g.

$$
Q_{x x}^{\prime}\left(q_{y}, 0\right)=-\tilde{K}_{2}\left(q_{y}\right) q_{y}^{2}
$$

this constant then depends on the wave vector

$$
\tilde{K}_{2}\left(q_{y}\right)=\frac{T q_{\mathrm{S}}^{2}}{16} \sqrt{\frac{M_{\mathrm{V}}}{M_{\mathrm{T}}}} \frac{1}{q_{y}} .
$$

The transition from the hydrodynamic limit to the temperature independent critical limit has already been observed experimentally by Delaye, Ribotta, and Durand [4] for the twist elastic constant $\widetilde{K}_{2}$, with $q_{z}=0$.

If the deviations $\delta \mathbf{n}$ of the director from the preferred axis $\mathbf{n}_{0}$ are not identified with external perturbations, the fluctuations of the director lead to corrections to the above results. As shown in Appendix $\mathrm{C}$ for the hydrodynamic limit, the correction term has the same critical temperature dependence as the above result, eq. (5.5). In view of the present experimental situation it seems unnecessary to calculate these corrections exactly.
6. The twist viscosity. - From the definition of the twist viscosity $\gamma_{1}$, eq. (3.13), we find directly

$$
\tilde{\gamma}_{1}(\mathbf{q}, \omega)=\frac{1}{\omega} \widetilde{Q}_{x x}^{\prime \prime}(\mathbf{q}, \omega),
$$

and with eq. (3.8)

$$
\tilde{\gamma}_{1}(\mathbf{q}, \omega)=\frac{1}{2 T}\left\langle\tilde{h}_{1 x}(\mathbf{q}, \omega) \tilde{h}_{1 x}(-\mathbf{q},-\omega)\right\rangle .
$$

This relation may be regarded as the Kubo formula for the twist viscosity. In the hydrodynamic limit $\mathbf{q} \rightarrow 0$ and $\omega \rightarrow 0$, we get with eq. (3.11), and the definition $\tilde{\gamma}_{1}(0,0) \equiv \tilde{\gamma}_{1}$,

$$
\tilde{\gamma}_{1}=\frac{q_{\mathrm{S}}^{2}}{2 T M_{\mathrm{T}}^{2}} \int \frac{\mathrm{d} \mathbf{K}}{(2 \pi)^{3}} K_{x}^{2}\left\langle|\psi(\mathbf{K})|^{2}\right\rangle^{2} \frac{1}{\Gamma(\mathbf{K})}
$$

and with eq. (2.5) and (2.9)

$$
\tilde{\gamma}_{1}=\frac{2 T q_{\mathrm{s}}^{2} \tau_{\mathrm{m}}}{(2 \pi)^{3} \xi_{\|}} \int \mathrm{d} \mathbf{P} \frac{P_{x}^{2}}{\left(1+P^{2}\right)^{11 / 4}} .
$$

This result already shows the critical temperature dependence of $\tilde{\gamma}_{1}$

$$
\tilde{\gamma}_{1}(T) \sim \frac{\tau_{\mathrm{m}}(T)}{\xi_{\|}(T)} \sim \xi^{1 / 2}(T) \sim\left(T-T_{\mathrm{c}}\right)^{-0.33} .
$$

We calculated the numerical factor by approximating the exponent $\frac{3}{4}$ in $\Gamma(\mathbf{K})$ by 1 . This leads to the result

$$
\tilde{\gamma}_{1}=\frac{T q_{\mathrm{S}}^{2} \tau_{\mathrm{m}}}{16 \pi \xi_{\|}}
$$

This expression is the exact analogue of the critical part of the static electrical conductivity in superconductors [6].

For a numerical estimate one may use [7]

$$
\tau_{\mathrm{m}}^{-1}=\frac{T-T_{\mathrm{c}}}{T_{\mathrm{c}}} \frac{1}{d^{2}} \sqrt{\frac{K_{1}}{\rho}}
$$

where $\rho$ is the mass density, to give

$$
\begin{aligned}
\tilde{\gamma}_{1}=\frac{k_{\mathrm{B}} T}{d^{2}} \sqrt{\frac{\rho d}{K_{1}}} \xi^{1 / 2} & \approx \\
& \approx 10^{-2}\left(\frac{T}{T-T_{\mathrm{c}}}\right)^{0.33} \text { poise },
\end{aligned}
$$

taking $K_{1} \approx 10^{-6} \mathrm{dyn}, d \approx 20 \AA, \rho \approx 1 \mathrm{~g} \mathrm{~cm}^{-3}$.

This effect can only be observed in a temperature region very close to $T_{\mathrm{c}}$. A value of $\tilde{\gamma}_{1}=0.1$ poise corresponds to $\Delta T=0.3^{\circ} \mathrm{C}$. 
In the mean field approximation, eq. (6.6) still holds but with

$$
\begin{aligned}
\tau_{\mathrm{m}} & \sim\left(T-T_{\mathrm{c}}\right)^{-1} \sim \xi^{2}(T) \\
\tilde{\gamma}_{1} & \sim \xi(T) \sim\left(T-T_{\mathrm{c}}\right)^{-0.5} .
\end{aligned}
$$

In the general case of arbitrary $q$ values, eq. (6.1) together with eq. (3.11) can still be used as dynamical scaling holds for the dynamical fluctuations of the order parameter, eq. (2.12) and (2.9). But the integration is more difficult here than in the case of the elastic constants. We restrict ourselves to the critical limit $q \xi \gg 1$, where we find

$$
\tilde{\gamma}_{1}(\mathbf{q}, 0) \sim \frac{\tau_{\mathrm{m}}}{\xi_{\|}} \int \mathrm{d} \mathbf{P} \frac{P_{x}^{2}}{\left(\mathbf{P}-\frac{\mathbf{q}}{2}\right)^{2}\left(\mathbf{P}+\frac{\mathbf{q}}{2}\right)^{2}\left[\left|\mathbf{P}-\frac{\mathbf{q}}{2}\right|^{3 / 2}+\left|\mathbf{P}+\frac{\mathbf{q}}{2}\right|^{3 / 2}\right]}
$$

Substituting $\mathbf{P}=\hat{q} \mathbf{Q}$ and $\hat{\mathbf{q}}=\hat{q} \mathbf{n}$ leads us to

$$
\tilde{\gamma}_{1}(\mathbf{q}, 0) \sim \frac{\tau_{\mathrm{m}}}{\xi_{\|}} \hat{q}^{-1 / 2} \int \mathrm{d} \mathbf{Q} \frac{Q_{x}^{2}}{\left(\mathbf{Q}-\frac{\mathbf{n}}{2}\right)^{2}\left(\mathbf{Q}+\frac{\mathbf{n}}{2}\right)^{2}\left[\left|\mathbf{Q}-\frac{\mathbf{n}}{2}\right|^{3 / 2}+\left|\mathbf{Q}+\frac{\mathbf{n}}{2}\right|^{3 / 2}\right]}
$$

The integral is independent of $q$. Therefore we get

$$
\tilde{\gamma}_{1}(q, 0) \sim q^{-1 / 2} .
$$

This result corresponds to eq. (5.10) for elastic constants, and shows also no critical temperature dependence. Combined with eq. (5.10) it yields for the critical spectrum of the director modes $\omega_{\mathrm{S}}$

$$
\omega_{\mathrm{S}} \sim \frac{\widetilde{K}(q, 0)}{\widetilde{\gamma}_{1}(q, 0)} q^{2} \sim q^{3 / 2}
$$

This result justifies the extension of dynamical scaling to the director modes, as supposed by F. Brochard [7].

The case of finite frequencies $\omega$, but $q \rightarrow 0$, may also be of some interest. In the approximation of a $q$ independent relaxation time $\Gamma^{-1}(\mathbf{K})=\tau_{\mathrm{m}}$ the result of eq. (6.1) and (3.11) is simply

$$
\tilde{\gamma}_{1}(0, \omega)=\frac{\tilde{\gamma}_{1}}{1+\left(\omega \tau_{\mathrm{m}}\right)^{2}}
$$

which shows the usual Landau-Khalatnikov behaviour for viscosities due to slowly relaxing internal processes.

As in the case of the elastic constants the fluctuations of the director can be shown to give corrections which do not alter the critical behaviour of $\tilde{\gamma}_{1}$ (see Appendix C).

7. Other viscosities. - As a complete set of viscosities for an incompressible nematic we use the LeslieParodi coefficients, called $\alpha_{i}$ and $\gamma_{i}$ in the notation of the Orsay Liquid Crystal Group [8]. We repeat their definition by giving the relations between the forces and fluxes. The forces in this case are the dissipative part $\sigma_{i j}^{\prime}$ of the stress tensor $\sigma_{i j}$ defined by

$$
\rho \frac{\partial v_{i}}{\partial t}=\nabla_{j} \sigma_{j i}
$$

and the dissipative part $\mathbf{h}^{\prime}$ of the molecular field $\mathbf{h}$. The fluxes are

$$
A_{i j}=\frac{1}{2}\left(\nabla_{i} \dot{v}_{j}+\nabla_{j} v_{i}\right)
$$

and

$$
\mathbf{N}=\frac{\partial \mathbf{n}}{\partial t}-\frac{1}{2}(\nabla \wedge \mathbf{v}) \wedge \mathbf{n}
$$

where $\mathbf{v}$ is the local velocity. Then we have

$$
\begin{aligned}
\sigma_{x x}^{\prime} & =\alpha_{4} A_{x x} \\
\sigma_{y y}^{\prime} & =\alpha_{4} A_{y y} \\
\sigma_{x y}^{\prime} & =\sigma_{y x}^{\prime}=\frac{1}{2} \alpha_{4} A_{x y} \\
\sigma_{z z}^{\prime} & =\left(\alpha_{1}+\alpha_{2}+\alpha_{3}+\alpha_{4}+2 \alpha_{5}\right) A_{z z} \\
\sigma_{x z}^{\prime} & =\left(\alpha_{2}+\alpha_{3}+\alpha_{4}+\alpha_{5}\right) A_{x z}+\alpha_{3} N_{x} \\
\sigma_{z x}^{\prime} & =\left(\alpha_{4}+\alpha_{5}\right) A_{z x}+\alpha_{2} N_{x} \\
\sigma_{y z}^{\prime} & =\left(\alpha_{2}+\alpha_{3}+\alpha_{4}+\alpha_{5}\right) A_{y z}+\alpha_{3} N_{y} \\
\sigma_{z y}^{\prime} & =\left(\alpha_{4}+\alpha_{5}\right) A_{z y}+\alpha_{2} N_{y}
\end{aligned}
$$

and

$$
\begin{aligned}
& h_{x}^{\prime}=\gamma_{1} N_{x}+\gamma_{2} A_{x z} \\
& h_{y}^{\prime}=\gamma_{1} N_{y}+\gamma_{2} A_{y z}
\end{aligned}
$$

with

$$
\gamma_{1}=\alpha_{3}-\alpha_{2}, \quad \gamma_{2}=\alpha_{3}+\alpha_{2} .
$$

In the special case $\mathbf{v}=0$, eq. (7.4) reduce to the dissipative part of eq. (3.13) used above.

Kubo formulas for the $\alpha_{i}$ 's are easily derived using the results of either linear response theory as done above, or the Landau-Lifshitz theory of fluctuations as done by Jähnig and $\mathrm{H}$. Schmidt [9] for another set of nematic viscosities. The result is, in the hydrodynamic limit $\mathbf{q} \rightarrow 0$ and $\omega \rightarrow 0$, 


$$
\begin{aligned}
& \tilde{\alpha}_{2}=\frac{1}{2 T}\left\langle\tilde{\sigma}_{z x}(0,0) \tilde{h}_{x}(0,0)\right\rangle \\
& \tilde{\alpha}_{3}=\frac{1}{2 T}\left\langle\tilde{\sigma}_{x z}(0,0) \tilde{h}_{x}(0,0)\right\rangle \\
& \tilde{\alpha}_{4}=\frac{1}{2 T}\left\langle\tilde{\sigma}_{x x}(0,0) \tilde{\sigma}_{x x}(0,0)\right\rangle \\
& \tilde{\alpha}_{4}+\tilde{\alpha}_{5}=\frac{1}{2 T}\left\langle\tilde{\sigma}_{z x}(0,0) \tilde{\sigma}_{z x}(0,0)\right\rangle \\
& \tilde{\alpha}_{1}+\tilde{\alpha}_{2}+\tilde{\alpha}_{3}+\tilde{\alpha}_{4}+2 \tilde{\alpha}_{5}= \\
&=\frac{1}{2 T}\left\langle\tilde{\sigma}_{z z}(0,0) \tilde{\sigma}_{z z}(0,0)\right\rangle .
\end{aligned}
$$

In order to apply these relations we need the fluctuating part $\tilde{\sigma}_{i j}$ of the stress tensor. According to the definition of the stress tensor, eq. (7.1), it is obtained from a variation of the free energy with respect to a small translation $\delta \mathbf{u}$ in space

$$
\delta F=-\int \mathrm{d} \mathbf{r} \nabla_{j} \sigma_{j i}^{t}(\mathbf{r}, t) \delta u_{i}(\mathbf{r}, t) .
$$

To carry out the variation of our free energy, eq. (2.1), we notice that the density $\rho^{\prime}(\mathbf{r}+\delta \mathbf{u})$ after the translation is equal to the density $\rho(\mathbf{r})$ before. Using the relation between the density and the order parameter [1]

$$
\rho(\mathbf{r})=\frac{1}{\sqrt{2}}\left(\mathrm{e}^{l q_{\mathrm{s}} z} \psi(\mathbf{r})+\text { c.c. }\right)
$$

we get

$$
\psi^{\prime}(\mathbf{r}+\delta \mathbf{u})=\psi(\mathbf{r}) \mathrm{e}^{-i q_{s} u_{z}}
$$

or

$$
\delta \psi(\mathbf{r})=-i q_{\mathrm{S}} \psi(\mathbf{r}) \delta u_{z}-\nabla_{i} \psi(\mathbf{r}) \delta u_{i} .
$$

The first term on the rhs is due to the smectic layered structure. The second term gives a negligible contribution to the critical elastic constants and viscosities, and will therefore be neglected. Using eq. (7.9) the variation of the free energy is straightforward and yields, with $\alpha=(x, y)$,

$$
\begin{aligned}
& \tilde{\sigma}_{\alpha z}(\mathbf{r}, t)=\frac{i q_{\mathrm{S}}}{2 M_{\mathrm{T}}}\left(\psi^{*}(\mathbf{r}, t) \nabla_{\alpha} \psi(\mathbf{r}, t)-\psi(\mathbf{r}, t) \nabla_{\alpha} \psi^{*}(\mathbf{r}, t)\right)+\frac{q_{\mathrm{S}}^{2}}{M_{\mathrm{T}}} \psi^{*}(\mathbf{r}, t) \psi(\mathbf{r}, t) \delta n_{\alpha}(\mathbf{r}, t) \\
& \widetilde{\sigma}_{z z}(\mathbf{r}, t)=\frac{i q_{\mathrm{S}}}{2 M_{\mathbf{V}}}\left(\psi^{*}(\mathbf{r}, t) \nabla_{z} \psi(\mathbf{r}, t)-\psi(\mathbf{r}, t) \nabla_{z} \psi^{*}(\mathbf{r}, t)\right) \\
& \widetilde{\sigma}_{l \alpha}(\mathbf{r}, t)=0
\end{aligned}
$$

A nonfluctuating contribution does not exist as we have restricted ourselves to an incompressible fluid. On comparison with eq. (3.2) we see that

$$
\tilde{\sigma}_{\alpha z}(\mathbf{r}, t)=-\widetilde{h}_{\alpha}(\mathbf{r}, t) .
$$

These relations permit us to evaluate the Kubo formulas without any further calculation. With eq. (7.12) we find

$$
\tilde{\alpha}_{2}=\tilde{\alpha}_{4}=\tilde{\alpha}_{5}=0
$$

and therefore, eq. (7.5),

$$
\tilde{\alpha}_{3}=\tilde{\gamma}_{2}=\tilde{\gamma}_{1}
$$

with $\tilde{\gamma}_{1}$ given by the above result eq. (6.6). Finally, comparing the Kubo formula eq. (7:6e) with the hydrodynamic limit of eq. (6.2) we obtain

$$
\tilde{\alpha}_{1}+\tilde{\alpha}_{3}=\left(\frac{M_{\mathrm{T}}}{M_{\mathrm{V}}}\right) \tilde{\gamma}_{1}
$$

or

$$
\tilde{x}_{1}=\frac{M_{\mathrm{T}}-M_{\mathrm{V}}}{M_{\mathrm{V}}} \widetilde{\gamma}_{1}
$$

A look at the viscosities which enter simple flow experiments may shed some insight on these results. In these measurements first done by Miesowitz [10] three different geometries are possible, as shown in figure 2. The corresponding viscosities are

$$
\begin{aligned}
& \eta_{1}=\frac{1}{2}\left(\alpha_{2}+2 \alpha_{3}+\alpha_{4}+\alpha_{5}\right) \\
& \eta_{2}=\frac{1}{2}\left(-\alpha_{2}+\alpha_{4}+\alpha_{5}\right), \quad \eta_{3}=\frac{1}{2} \alpha_{4} .
\end{aligned}
$$

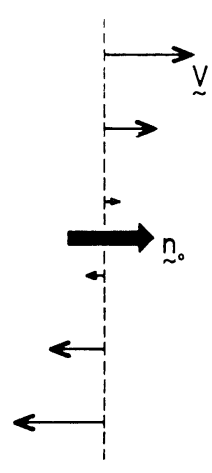

$\eta_{1}$

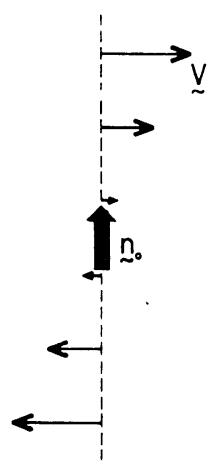

$\eta_{2}$

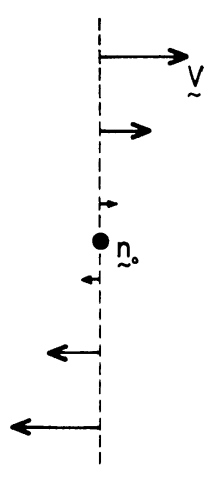

$\eta_{3}$
Fig. 2. - The three possible geometries for simple shear flou corresponding to Miesowicz' notation. 
Inserting our results, eq. (7.14) and (7.15), we get

$$
\begin{aligned}
& \tilde{\eta}_{1}=\tilde{\gamma}_{1} \\
& \tilde{\eta}_{2}=\tilde{\eta}_{3}=0 .
\end{aligned}
$$

These relations express the fact that only in case 1 the fluctuating layer structure is disturbed by the flow of the molecules.

8. Conclusion. - We have investigated the critical contribution to the elastic constants and the viscosities of a nematic above a nematic-smectic A transition of second order. We started from two distinct assumptions, which have already been introduced by de Gennes [1] to calculate the critical behaviour of the elastic constants: i) The fluctuations of the smectic order parameter are coupled to the director by means of a free energy expression of the Ginzburg-Landau type, in analogy to the case of charged superfluids, and ii) the nematic-smectic A transition is thermodynamically similar to the $\lambda$-transition in $\mathrm{He}^{4}$, an uncharged superfluid for which mean field theory does not hold; therefore the critical exponents for the dynamical fluctuations of the order parameter have to be taken according to the general lines of scaling theory [7].

Assumption i) implies the ínvariance of the system under a simultaneous local rotation of the director and the smectic layers in the cybotactic groups (except for splay terms). As a consequence of this smectic gauge invariance the director response function has to be transverse. In our calculation we worked only in lowest order of the (scaling invariant) order parameter fluctuations, but ensured that our director response function is transverse. As scaling holds for the order parameter fluctuations we are able to treat the general case of arbitrary wave num- bers. In the hydrodynamic limit we find again de Gennes' [1] result $\widetilde{K}_{2} \sim \widetilde{K}_{3} \sim\left(T-T_{\mathrm{c}}\right)^{-0.66}$ which has been verified recently [2]-[4], and for the critical viscosities $\tilde{\gamma}_{1}, \tilde{\gamma}_{2}, \tilde{\alpha}_{1}$, and $\tilde{\alpha}_{3}$ we predict a divergence $\left(T-T_{\mathrm{c}}\right)^{-0.33}$. The latter result may be investigated experimentally by the same methods which have been successfully used to determine ordinary viscosities in nematics :

i) classical flow measurements [10],

ii) determination of flow-alignment angle [11],

iii) rotating magnetic field [12],

iv) dynamical Frederiks transition [13],

v) quasielastic scattering of light [14], and

vi) shear wave reflection [15].

In the transition region from the hydrodynamic to the critical regime we derived, a temperature dependence of the static response function which fits well the experimental result [4]. In the critical regime the wave number dependent elastic constants and viscosities behave like $q^{-1}$ and $q^{-1 / 2}$, respectively. The critical spectrum of the director modes is given by $\omega_{\mathrm{S}} \sim q^{3 / 2}$. As the critical spectrum of the order paramater fluctuations shows the same $q$ dependence [7] this result means that the director modes obey dynamical scaling, as already proposed by F. Brochard. In contrast to He, the $q$ dependence of the critical spectrum may be measured by light scattering experiments, as it was observed for planar antiferromagnets by neutron scattering [16].

Acknowledgment. - We would like very much to thank P. G. de Gennes for many illuminating discussions during the whole period when this work was done. One of us (F. J.) would also like to thank all the members of the Physique de la Matière condensée at the Collège de France for their kind hospitality during his stay in Paris.

\section{APPENDIX A.}

SMectic gauge InVARIANCE AND THE STATIC RESPONSE. - The static response function is according to eq. (3.12)

$$
Q_{\alpha \beta}^{\mathrm{d}}(\mathbf{q}, 0)=\frac{q_{\mathrm{S}}^{2}}{4 T M_{\mathrm{T}}^{2}} \int \frac{\mathrm{d} \mathbf{k}_{1}}{(2 \pi)^{3}}\left(k_{1}+k_{2}\right)_{\perp \alpha}\left(k_{1}+k_{2}\right)_{\perp \beta}\left\langle\left|\psi\left(\mathbf{k}_{1}\right)\right|^{2}\right\rangle\left\langle\left|\psi\left(\mathbf{k}_{2}\right)\right|^{2}\right\rangle
$$

where we have introduced

$$
\mathbf{k}_{1}=\mathbf{K}+\frac{\mathbf{q}}{2} \quad \text { and } \quad \mathbf{k}_{2}=\mathbf{K}-\frac{\mathbf{q}}{2}=\mathbf{k}_{1}-\mathbf{q}
$$

To show that the longitudinal part of $Q_{\alpha \beta}(\mathbf{q}, 0)$ vanishes we calculate

$$
q_{\beta} Q_{\alpha \beta}^{\mathrm{d}}(\mathbf{q}, 0)=\frac{q_{\mathrm{S}}^{2}}{2 T M_{\mathrm{T}}} \int \frac{\mathrm{d} \mathbf{k}_{1}}{(2 \pi)^{3}}\left(k_{1}+k_{2}\right)_{\perp \alpha} \frac{k_{1 \perp}^{2}-k_{2 \perp}^{2}}{2 M_{\mathrm{T}}}\left\langle\left|\psi\left(\mathbf{k}_{1}\right)\right|^{2}\right\rangle\left\langle\left|\psi\left(\mathbf{k}_{2}\right)\right|^{2}\right\rangle .
$$


If we add to the fractional term the term $\frac{k_{1 z}^{2}-k_{2 z}^{2}}{2 M_{\mathrm{V}}}$, whose contribution is zero, we can perform a fractional analysis using eq. (2.5)

$$
q_{\beta} Q_{\alpha \beta}^{\mathrm{d}}(\mathbf{q}, 0)=\frac{q_{\mathrm{S}}^{2}}{2 M_{\mathrm{T}}} \int \frac{\mathrm{d} \mathbf{k}_{1}}{(2 \pi)^{3}}\left(k_{1}+k_{2}\right)_{\perp \alpha}\left[\left\langle\left|\psi\left(\mathbf{k}_{2}\right)\right|^{2}\right\rangle-\left\langle\left|\psi\left(\mathbf{k}_{1}\right)\right|^{2}\right\rangle\right]
$$

As

$$
\begin{aligned}
\int \frac{\mathrm{d} \mathbf{k}_{1}}{(2 \pi)^{3}}\left(k_{1}+k_{2}\right)_{\perp \alpha}\left\langle\left|\psi\left(\mathbf{k}_{1}\right)\right|^{2}\right\rangle & =\int \frac{\mathrm{d} \mathbf{k}_{1}}{(2 \pi)^{3}}\left(2 k_{1}-q\right)_{\perp \alpha}\left\langle\left|\psi\left(\mathbf{k}_{1}\right)\right|^{2}\right\rangle \\
& =-q_{\alpha}\left\langle|\psi|^{2}\right\rangle
\end{aligned}
$$

and analogously for the other part of the integral, we find the result

$$
q_{\beta} Q_{\alpha \beta}^{\mathrm{d}}(\mathbf{q}, 0)=\frac{q_{\mathrm{s}}^{2}}{M_{\mathrm{T}}}\left\langle|\psi|^{2}\right\rangle q_{\alpha} .
$$

With eq. (3.4) and (3.5), this yields

$$
q_{\beta} \widetilde{Q}_{\alpha \beta}(\mathbf{q}, 0)=0
$$

\section{APPENDIX B.}

The StATIC ReSPONSE FUnCtion. - We want to calculate the elastic response $Q_{x x}^{\prime}(\mathbf{q}, 0)$ for $q_{x}=0$. From eq. (4.6) follows

$$
Q_{x x}^{\prime}(\mathbf{q}, 0)=Q_{x x}^{\mathrm{d}^{\prime}}(\mathbf{q}, 0)-Q_{x x}^{\mathrm{d}^{\prime}}(0,0)
$$

$Q_{x x}^{\mathrm{d}^{\prime}}(\mathbf{q}, 0)$ is given by eq. $(5.2)$

$$
Q_{x x}^{\mathrm{d}^{\prime}}(\mathbf{q}, 0)=\frac{4 T q_{\mathrm{S}}}{(2 \pi)^{3} \xi_{\|}} J(\hat{\mathbf{q}})
$$

with

$$
J(\hat{\mathbf{q}})=\int \mathrm{d} \mathbf{P} \frac{P_{x}^{2}}{\left[1+\left(\mathbf{P}+\frac{\hat{\mathbf{q}}}{2}\right)^{2}\right]\left[1+\left(\mathbf{P}-\frac{\hat{\mathbf{q}}}{2}\right)^{2}\right]}
$$

To evaluate this integral analytically we use a trick introduced in field theory by Feynman

$$
\frac{1}{A B}=\int_{0}^{1} \mathrm{~d} t \frac{1}{[t A+(1-t) B]^{2}}
$$

Application of this formula to $J(\hat{\mathbf{q}})$ yields

$$
\begin{aligned}
J(\hat{\mathbf{q}}) & =\int_{0}^{1} \mathrm{~d} t \int \mathrm{d} \mathbf{P} \frac{P_{x}^{2}}{\left[\left(\mathbf{P}+\left(t-\frac{1}{2}\right) \hat{\mathbf{q}}\right)^{2}+1+t(1-t) \hat{q}^{2}\right]^{2}} \\
& =\frac{1}{3} \int_{0}^{1} \mathrm{~d} t \int \mathrm{d} \mathbf{P}^{\prime} \frac{P^{\prime 2}}{\left[P^{\prime 2}+1+t(1-t) \hat{q}^{2}\right]^{2}}
\end{aligned}
$$


because of $q_{x}=0$. To eliminate the divergence of the integral in the expression (B.1) we use the following relation

$$
\begin{aligned}
\int \mathrm{d} \mathbf{P} \frac{P^{2}}{\left[1+\left(\mathbf{P}+\frac{\hat{\mathbf{q}}}{2}\right)^{2}\right]\left[1+\left(\mathbf{P}-\frac{\hat{\mathbf{q}}}{2}\right)^{2}\right]} & = \\
& =\int \mathrm{d} \mathbf{P} \frac{1}{1+P^{2}}-\left(1+\frac{\hat{q}^{2}}{4}\right) \int \mathrm{d} \mathbf{P} \frac{1}{\left[1+\left(\mathbf{P}+\frac{\hat{\mathbf{q}}}{2}\right)^{2}\right]\left[1+\left(\mathbf{P}-\frac{\hat{\mathbf{q}}}{2}\right)^{2}\right]}
\end{aligned}
$$

which can easily be verified. On the rhs the divergence is contained in the first term, which is $q$ independent. On the other hand, the relation between the expression on the lhs and $J(\hat{q})$ is found, again using eq. (B.4), to be

$$
\begin{aligned}
\int \mathrm{d} P \frac{P^{2}}{\left[1+\left(\mathbf{P}+\frac{\hat{\mathbf{q}}}{2}\right)^{2}\right]\left[1+\left(\mathbf{P}-\frac{\hat{\mathbf{q}}}{2}\right)^{2}\right]} & \\
& =3 J(\hat{\mathbf{q}})+\frac{\hat{q}^{2}}{4} \int_{0}^{1} \mathrm{~d} t(2 t-1)^{2} \int \mathrm{d} \mathbf{P}^{\prime} \frac{1}{\left[P^{\prime 2}+1+t(1-t) \hat{q}^{2}\right]^{2}} .
\end{aligned}
$$

Combining eq. (B.6) and (B.7) we get

$$
\begin{aligned}
& J(\hat{\mathbf{q}})=\frac{1}{3}\left\{\int \mathrm{d} \mathbf{P} \frac{1}{1+P^{2}}-\left(1+\frac{\hat{q}^{2}}{4}\right) \int_{0}^{1} \mathrm{~d} t \int \mathrm{d} \mathbf{P}^{\prime} \frac{1}{\left[P^{\prime 2}+1+t(1-t) \hat{q}^{2}\right]^{2}}-\right. \\
& \left.-\frac{\hat{q}^{2}}{4} \int_{0}^{1} \mathrm{~d} t(2 t-1)^{2} \int \mathrm{d} \mathbf{P}^{\prime} \frac{1}{\left[P^{\prime 2}+1+t(1-t) \hat{q}^{2}\right]^{2}}\right\} .
\end{aligned}
$$

The momentum integration in the $\hat{q}$ dependent terms can now easily be done, and we obtain

$$
J(\hat{\mathbf{q}})=\frac{1}{3}\left\{\int \mathrm{d} \mathbf{P} \frac{1}{1+P^{2}}-\pi^{2} \int_{0}^{1} \mathrm{~d} t \frac{1}{\sqrt{1+t(1-t) \hat{q}^{2}}}\left[1+\frac{\hat{q}^{2}}{2}\left(2 t^{2}-2 t+1\right)\right]\right\}
$$

and therefore

$$
J(\hat{\mathbf{q}})-J(0)=-\frac{\pi^{2}}{3} \int_{0}^{1} \mathrm{~d} t\left[\frac{1+\frac{\hat{q}^{2}}{2}\left(2 t^{2}-2 t+1\right)}{\sqrt{1+t(1-t) \hat{q}^{2}}}-1\right] .
$$

Performing the remaining integration we find the result eq. (5.7).

\section{APPENDIX C.}

DireCTOR FLUCTUATIONS. - If we want to take into account the fluctuations of the director we have to use

$$
\delta \mathbf{n}(\mathbf{r}, t)=\delta \widetilde{\mathbf{n}}(\mathbf{r}, t)+\delta \mathbf{n}^{\mathrm{ext}}(\mathbf{r}, t),
$$

where $\delta \tilde{\mathbf{n}}(\mathbf{r}, t)$ represents the fluctuations. In section 2 we treated the case $\delta \tilde{\mathbf{n}}(\mathbf{r}, t)=0$. To calculate the response function $Q_{\alpha \beta}^{\mathrm{n}}(\mathbf{q}, \omega)$ due to fluctuations $\delta \tilde{\mathbf{n}}(\mathbf{r}, t)$ we can still use eq. (3.6) and (3.7). We treat only the imaginary part and find

$$
Q_{\alpha \beta}^{\mathrm{n}^{\prime \prime}}(\mathbf{q}, \omega)=\frac{\omega}{2 T}\left\langle\widetilde{h}_{2 \alpha}(q, \omega) \widetilde{h}_{2 \beta}(-\mathbf{q},-\omega)\right\rangle
$$


with

$$
\begin{aligned}
& \tilde{\mathbf{h}}_{2}(\mathbf{q}, \omega)= \\
& \quad=-\frac{q_{\mathrm{S}}^{2}}{M_{\mathrm{T}}} \int \frac{\mathrm{d} \mathbf{K} \mathrm{d} \mathbf{K}^{\prime} \mathrm{d} \Omega \mathrm{d} \Omega^{\prime}}{(2 \pi)^{8}} \psi^{\prime}\left(\mathbf{K}-\frac{\mathbf{q}}{2}, \Omega-\frac{\omega}{2}\right) \psi^{\prime}\left(\mathbf{K}^{\prime}+\frac{\mathbf{q}}{2}, \Omega^{\prime}+\frac{\omega}{2}\right) \delta \tilde{\mathbf{n}}_{t}\left(\mathbf{K}-\mathbf{K}^{\prime}, \Omega-\Omega^{\prime}\right)
\end{aligned}
$$

where we have used the fact that we can eliminate the longitudinal part $\delta \tilde{\mathbf{n}}_{l}(\mathbf{r}, t)$ of the director from the free energy and the molecular field expressions, and take $\psi^{\prime}(\mathbf{r})$ real (see section 4$)$. Then, in the thermal average of eq. (C. 1), we may decouple the fast motion of the modulus of the order parameter and the hydrodynamic motion of the director $\delta \tilde{\mathbf{n}}_{t}$, in analogy to the decoupling scheme applied to binary mixtures by Ferrell [17]. Within this decoupling procedure the cross terms $\left\langle\widetilde{h}_{1 \alpha}(\mathbf{q}, \omega) \widetilde{h}_{2 \beta}(-\mathbf{q},-\omega)\right\rangle$ give no contribution. The contribution to the rotational viscosity can then be written as

$$
\begin{aligned}
\tilde{\gamma}_{1}^{\mathrm{n}} & =\frac{q_{\mathrm{S}}^{4}}{2 T M_{\mathrm{T}}^{2}} \int \frac{\mathrm{d} \mathbf{K} \mathrm{d} \mathbf{K}^{\prime} \mathrm{d} \Omega}{(2 \pi)^{7}}\left\langle\left|\psi^{\prime}(\mathbf{K}, \Omega)\right|^{2}\right\rangle\left\langle\left|\psi^{\prime}\left(\mathbf{K}^{\prime}, \Omega\right)\right|^{2}\right\rangle\left\langle\left|\delta \tilde{n}_{t x}\left(\mathbf{K}-\mathbf{K}^{\prime}\right)\right|^{2}\right\rangle \\
& =\frac{q_{\mathrm{S}}^{4}}{T M_{\mathrm{T}}^{2}} \int \frac{\mathrm{d} \mathbf{K} \mathrm{d} \mathbf{K}^{\prime}}{(2 \pi)^{6}}\left\langle\left|\psi^{\prime}(K)\right|^{2}\right\rangle\left\langle\left|\psi^{\prime}\left(K^{\prime}\right)\right|^{2}\right\rangle \frac{1}{\Gamma(\mathbf{K})+\Gamma\left(\mathbf{K}^{\prime}\right)}\left\langle\left|\delta \tilde{n}_{t x}\left(\mathbf{K}-\mathbf{K}^{\prime}\right)\right|^{2}\right\rangle
\end{aligned}
$$

The thermal average $\left\langle\left|\delta n_{t x}(\mathbf{K})\right|^{2}\right\rangle$ can easily be derived from the relation [8]

$$
\delta \mathbf{n}_{t}(\mathbf{K})=\delta \mathbf{n}_{1}(\mathbf{K})+\delta \mathbf{n}_{2}(\mathbf{K})-\delta \mathbf{n}_{l}(\mathbf{K})=\left(\mathbf{e}_{1}-\frac{\left(\mathbf{e}_{1} \cdot \mathbf{K}\right) \mathbf{K}}{K^{2}}\right) n_{1}(\mathbf{K})+\mathbf{e}_{2} n_{2}(\mathbf{K})
$$

as

$$
\left\langle\left|\delta \tilde{n}_{t x}(\mathbf{K})\right|^{2}\right\rangle=\cos ^{2} \varphi \cos ^{4} \theta\left\langle\left|\delta \tilde{n}_{1}(\mathbf{K})\right|^{2}\right\rangle+\sin ^{2} \varphi\left\langle\left|\delta \tilde{n}_{2}(\mathbf{K})\right|^{2}\right\rangle
$$

with [7]

$$
\begin{aligned}
& \left\langle\left|\delta \tilde{n}_{1}(\mathbf{K})\right|^{2}\right\rangle=\frac{T}{K_{1} q_{\perp}^{2}+K_{3}^{t} q_{z}^{2}} \\
& \left\langle\left|\delta \tilde{n}_{2}(\mathbf{K})\right|^{2}\right\rangle=\frac{T}{K_{2}^{t} q_{\perp}^{2}+K_{3}^{t} q_{z}^{2}} .
\end{aligned}
$$

The angles $(\varphi, \theta)$ are the polar coordinates of $\mathbf{K}$, where $\theta=0$ is the $z$ direction. Taking the full elastic constants eq. (C.6) corresponds to the use of the renormalized propagator for the director field.

As the integral in eq. (C.3) could not be solved analytically we deduce its temperature dependence by neglecting the $K$ dependence of $\left\langle\left|\psi^{\prime}(\mathbf{K})\right|^{2}\right\rangle$ and $\Gamma(\mathbf{K})$ and integrating up to $\xi^{-1}$. This yields

$$
\tilde{\gamma}_{1}^{\mathrm{n}} \sim \frac{T^{2} q_{\mathrm{S}}^{4} \tau_{\mathrm{m}}}{\widetilde{K}_{2,3}}
$$

Inserting the result for $K_{2,3}$, eq. (5.5), we get

$$
\tilde{\gamma}_{1}^{\mathrm{n}} \sim \frac{T q_{\mathrm{S}}^{2} \tau_{\mathrm{m}}}{\xi} \sim \tilde{\gamma}_{1}
$$

This result shows that the fluctuations of the director give an additional contribution to the rotational viscosity with the same critical temperature dependence as $\tilde{\gamma}_{1}$. Analogously, it is shown that $\widetilde{K}_{2,3}^{\mathrm{n}} \sim \widetilde{K}_{2,3}$.

\section{References}

[1] De Gennes, P. G., Solid State Commun. 10 (1972) 753.

[2] Cheung, L., Meyer, R. B., Gruler, H., Phys. Rev. Lett. 31 (1973) 349.

[3] LÉGer, L., Phys. Lett. 44A (1973) 535.

[4] Delaye, M., Ribotta, R., Durand, G., Phys. Rev. Lett. 31 (1973) 443.

[5] Schmid, A., Phys. Rev. 180 (1969) 527.
[6] Schmidt, H., Z. Phys. 216 (1968) 336.

[7] Brochard, F., J. Physique 34 (1973) 411.

[8] Orsay Liquid Crystal Group, J. Chem. Phys. 51 (1969) 816.

[9] Jähnig, F., Schmidt, H., Ann. Phys. (N. Y.) 71 (1972) 129. The viscosities used by them are related to the Leslie coefficients by : $\zeta_{1}=\gamma_{1}, v=-\gamma_{2}, \eta_{2}=\frac{1}{2} \alpha_{4}, \eta_{4}=\gamma_{2}$ $+2\left(\alpha_{4}+\alpha_{5}\right), \eta_{5}=\alpha_{1}+\alpha_{2}+\alpha_{3}+\alpha_{4}+2 \alpha_{5}$. 
[10] Miesowicz, M., Nature 158 (1946) 27.

[11] WaHL, J., Fisher, F., Opt. Commun. 5 (1972) 341. GäHWILLER, Ch., Phys. Rev. Lett. 28. (1972) 1554.

Меiвоom, S., Hewitt, R. C., Phys. Rev. Lett. 30 (1973) 261.

[12] Prost, J., Gasparoux, H., Phys. Lett. 36A (1971) 245.

[13] Brochard, F., Pieranski, P., Guyon, E., Phys. Rev. Lett. 28 (1972) 1681.

LÉGER, L., Solid State Commun. 11 (1972) 1499.

[14] Orsay Liquid Crystal Group, Mol. Cryst. Liquid Cryst. 13 (1971) 187.
[15] Martinoty, P. (*), Candau, S., Mol. Cryst. Liquid Cryst. 14 (1971) 243.

[16] Lau, H. Y., Corliss, L. M., Delapalme, A., Hastings, J. M., Nathans, R., Tucciarone, A., Phys. Rev. Lett. 23 (1969) 1225.

[17] Ferrell, R. A., Phys. Rev. Lett. 24 (1970) 1169.

FerRell, R. A., Dynamical aspects of critical phenomena, edited by J. I. Budnick and M. P. Kawatra (Gordon and Breach) 1970.

(*) Dr. Martinoty has pointed out to us that in shear wave reflection measurements a cancellation occurs and no critical effect is expected in this experiments. 